\title{
Tinnitus and stress in adults: A scoping review
}

\section{Introduction}

Tinnitus is the perception of sound in absence of any external acoustic stimulation, and is associated with otological and non-otological disorders (Baguley et al., 2013). Tinnitus signals are varied in loudness, pitch, and spectral contents (psychoacoustic characteristics) among those who experience it (Cederroth et al., 2019), which can impact the emotional and mental status of the person differently (Møller, 2016; Hall et al., 2018a). A relationship between tinnitus and stress is frequently reported in literature (including fiction), patient information leaflets, and online tinnitus information. Stress is a recognised risk factor for several systemic illnesses and psychological disorders such as heart diseases, depression, and anxiety potentially through changes in metabolic and immune activity, adoption of unhealthy behaviours, or alteration in neuronal networks involved in the regulation of emotion and cognition (Duric et al., 2016). Although it is not clear if stress is a risk factor for, or a consequence of tinnitus (Pattyn et al., 2016), changes in neuronal networks is a possible explanation for a relationship. Tinnitus perception is maintained by non-auditory neuronal circuits (Rauschecker et al., 2010). Excessive neuronal activity in the limbic system, para-limbic structure, frontal and temporal lobes are commonly found in tinnitus patients (Henry et al., 2014; Kreuzer et al., 2013). Similarly, these areas have shown stress-related structural and functional changes (Henry et al., 2014; Kreuzer et al., 2013). Cognitive functions are mainly regulated by different limbic structures such as prefrontal cortex, amygdala, and hippocampus (Rajmohan and Mohandas, 2007). Cognitive functions can be affected by stress (Boals and Banks, 2012; Fisher et al., 2017) and tinnitus (Tegg-Quinn et al., 2016; Clarke et al., 2020; Mohamad et al., 2016; Andersson and McKenna, 2006). Additionally, Stress-related psychological disorders, mainly anxiety and depression, are 
highly prevalent in tinnitus patients (Milerova et al., 2013; Crocetti et al., 2009; Pattyn et al., 2016; Pinto et al., 2014; Durai and Searchfield, 2016). Presumably, stress and tinnitus share many features and occur simultaneously, which suggests a relationship.

Although there is some evidence for a relationship between stress and tinnitus, there are still questions about the extent of the contribution of stress to tinnitus pathogenesis, and the direction of this relationship, which need to be clarified (Hébert et al., 2017). This is a complex area of research given the complexity of stress concept. For any given individual, stress can be defined as the feeling of mental and emotional strain, tension, and worry associated with a stressful or demanding event. In psychology, stress is defined as the biophysiological changes that occur in response to exposure to a demanding event (i.e. response-based concept) (Saxena, 1980; McEwen, 2005). These responses include activation and inhibition of several neuroendocrine axes, mainly the hypothalamic-pituitary-adrenal axis and sympathetic-adrenal-medullary axis. Both, in turn, regulate different metabolic and immunological functions like lipolysis and glycogenolysis (Schneiderman et al., 2005) through their end effectors, mainly cortisol, epinephrine (EPI), and norepinephrine (NE). Thus, stress can be measured by assessing the level of stress mediators and their physiological effects on the body (Rice, 2012; Saxena, 1980). Stress is also defined as an event that a person experiences which stimulates physical and psychological adaptive effects on the body, for example, jobrelated stress (Papathanasiou et al., 2015). This definition is the stimulus-based concept of stress in which stress is measured by the intensity and duration of the event, for example using the Daily Hassles Scale (Kanner et al., 1981), the Social Readjustment Rating Scale (Holmes and Rahe, 1967), or Interview-based assessment of life events (Monroe, 2008). The third definition of stress is the interaction between stressors (internal or external), an individual's perception and interpretation of the stressors, and 
the available resources for coping with the stressors. In other words, stress is modulated by cognition and coping (Lazarus and Folkman, 1984). This transactional model hypothesises that the key component in developing diseases is perceived stress, which is measured by different instruments such as the Perceived Stress Scale (PSS) (Cohen et al., 1983), Perceived Stress Questionnaire (PSQ) (Levenstein et al., 1993), or the Depression Anxiety Stress Scale (DASS) (Lovibond and Lovibond, 1995). Tinnitus can be measured by self-reported questionnaires (psychometric instruments) that assess "tinnitus reaction" and by psychoacoustic measurements that assess "tinnitus perception" (Henry et al., 2019). Therefore there are multiple different ways in which stress and tinnitus can be measured and assessed, which could in turn affect the supposed relationship between tinnitus and stress. Consequently, in order to build an understanding of the current position of the research in stress and tinnitus, there is a need to identify research evidence and gaps in existing knowledge on the relationship between tinnitus and stress in adults. The evidence could be used to improve clinical practice for tinnitus and identify whether stress management should be prioritised, and identify specific research questions and elements that should underpin the design of any new studies or systematic review.

When there is limited evidence on the current position of research on a topic (e.g. the relationship between tinnitus and stress), and the potential research gaps within the existing literature to know the value of undertaking a systematic review and to be able to form the precise research question needed for a systematic review, scoping reviews are often conducted as a precursor to a systematic review. Scoping review methodology enables broad research questions to be addressed, examining the extent of evidence across large and diverse range of topics and study designs, whilst still incorporating rigorous methodological practices throughout the process similar to that used in a 
systematic reviews (Peters et al., 2015). This scoping review methodology therefore allows us to undertake an inductive approach to conduct an in-depth exploration of the complex relationship between tinnitus and stress, taking into account the complexity of the stress concept and the variety of assessment instruments used from a broad range of study designs. Therefore, the aim of this scoping review is to catalogue research to date on the relationship between tinnitus and stress in adults (in all three concepts of stress described above), and specifically the contribution of stress to the prevalence of tinnitus, reported stress-related biophysiological changes in tinnitus patients, perceived stress levels in tinnitus patients, and the effect of tinnitus management on stress and vice versa.

\section{Methods}

This review was conducted and reported in accordance to scoping review protocol guidelines by Arksey and O'Malley (2005) and Levac et al. (2010). As per guidelines, we followed a five-stage framework for the conduct of scoping reviews. This involve (i) identifying the review question, (ii) identifying the relevant studies, (iii) study selection, (iv) charting the data, (v) collating, summarising and reporting the results. This method is in accordance with the PRISMA-ScR checklist for scoping reviews (Tricco et al., 2018).

\section{Stage i: Identifying the question}

The key question of this scoping review is "What is known about the relationship between stress and tinnitus?" It was formed to guide the search and identify the inclusion criteria. 


\section{Stage ii: Identifying the relevant studies}

\section{Eligibility criteria}

The inclusion criteria were (1) original articles published in English, (2) conducted in adult humans (18 years and above, no upper age limit), and (3) both tinnitus and stress were measured separately as in a specific construct of stress was measured and this was independent of measurements of the construct tinnitus symptom severity (which may contain some items referring to tinnitus-related stress). The last inclusion criteria was essential to compare levels of stress with level of tinnitus. There are various classification systems for tinnitus such as primary vs secondary, and subjective vs objective. All classifications of tinnitus were eligible in this review.

Review articles including systematic reviews, epidemiology articles, and any sources reporting personal/expert opinions were excluded. Records relating to basic scientific research in animals, Posttraumatic Stress Disorder (PTSD), trauma, and stress reactivity (oxidative stress) were also excluded. Where multiple eligible unique records pertaining to a single trial were identified, the record that was published first was included and any secondary analyses of the data were excluded.

\section{Search strategy}

Literature searches were undertaken in relevant electronic databases, namely PsycINFO (OVID), Medline (OVID), EMBASE (OVID), PILOTS, CINAHL, Web of Science, Pubmed, Scopus and Google scholar. ProQuest Dissertations and Theses, and Nottingham eTheses and EThOS were searched for grey literature. The search strategy varied by database (example in Table 1). 
Using the search terms tinnitus AND stress* AND adult AND human, the searches for each term were conducted separately and then search results were combined. When possible, the search was limited to English. The search was not limited to any specific date. Additionally, reference lists of any review articles from the electronic search and the table of contents of the last 6 months of key journals were hand-searched for potentially relevant articles. The initial searches were conducted in October 2017. The final electronic search was conducted in July 2019 and hand-search in November 2019.

\section{Stage iii: Study selection}

Records identified through electronic and hand searches were exported into Endnote and duplicates were removed. Study selection had two stages. In the first stage, titles and abstracts were screened independently by two reviewers, one of those is the primary author (AE) and one of the co-authors (KF, DB, or DH). Disagreements between reviewers resolved through discussion. All records that held the possibility of relevant findings were retained for full-text screening. Full texts were screened independently by two reviewers against the inclusion/exclusion criteria. Disagreements were resolved through discussions or the involvement of a third reviewer.

\section{Stage iv: Charting the data}

A data extraction form was developed in Microsoft Excel and modified following piloting with two included studies. Two reviewers (AE, the primary author, with KF, $\mathrm{DB}$, or $\mathrm{DH})$ extracted data from each record independently and compared the results. Any disagreements in the extracted data were resolved through discussion and revisiting the record. Data was extracted on study identifiers (authors' names, date of publication, title, and type of the study), study characteristics (aims, sample size, participants age, presence of hearing loss and tinnitus) assessment instruments used to measure tinnitus 
and stress, and any reported relationship between tinnitus and stress as reported in the findings of the study.

\section{Stage v: Collating, summarizing and reporting results}

The extracted data on the study characteristics and assessment instruments were initially summarised and tabulated. Further to this, the information related to the assessment instruments, results of the study and the relationship between tinnitus and stress was subjected to thematic analysis in order to catalogue and summarize similar findings into four themes: assessment instruments used, evidence of a relationship, biomarkers of stress in tinnitus, and changes after an intervention.

\section{Results}

Initial database searches produced 4911 records. After removing duplicates, title, and abstract screening, 131 records were selected for full-text screening. Following full-text screening, an additional 93 records were excluded, leaving 38 included records retrieved for data extraction. From our updated literature searches in July 2019 and November 2019, a further 12 records were identified as eligible for data extraction (Figure 1). Studies were in general excluded because stress was not measured, or they included participants younger than 18 years old. Details of the study design, age of the participants, and tinnitus and stress measurements summarised in Supplementary Table.

\section{$<$ Figure 1>}

Hearing was assessed in 32 studies. The other 19 studies did not report whether hearing was assessed or not. Audiometric tests were used in 18 studies (mainly pure tone audiometry). Eight studies used self-report instruments (i.e. participants answered 
questions about hearing impairment) and reported the percentages of participants who had hearing impairment. Only five studies measured hearing but did not report the results. Few studies were designed to examine tinnitus associated with hearing impairment. Those that were had specific populations who had either sudden sensorineural hearing loss (Schmitt et al., 2000), single sided deafness (Haussler et al., 2019), or used cochlear implants (Olze et al., 2012; Olze et al., 2011a; Olze et al., 2011b; Ketterer et al., 2018) (see also Supplementary Table).

Among included studies, only 25 were specifically designed to assess stress within tinnitus population. The aims were to assess psychological and biophysiological stress in tinnitus patients, to describe the modulating factors of the relationship between tinnitus and stress, to assess the effect of management on tinnitus and stress, to measure the occurrence of stress in tinnitus patients, or to evaluate the effect of stressful life events on tinnitus patients. The remaining 18 studies had different aims that included testing the effect of tinnitus managements on tinnitus, describing tinnitus features in a group of tinnitus patients, tinnitus questionnaire validation, and others.

\section{Measurements used in the included studies}

The most frequently used standardised stress instruments in all included studies were the PSQ, stress Visual Analogue Scales (VAS), and the DASS which are perceived stress measurements. Stimulus-based stress measurements were used in three studies. Biophysiological markers of stress were measured in 15 studies. Four studies assessed the occurrence of stress symptoms among people who have tinnitus. Only two studies assessed job-related stress (Abbott et al., 2009; Saruhanoglu et al., 2017).

Tinnitus symptom severity was measured in all studies; in most of them two instruments were used. The most frequently used instruments were VAS, the Tinnitus 
Handicap Inventory (THI) (Newman et al., 1996), the Tinnitus Questionnaire (TQ) (Hallam et al., 1988), or the Tinnitus Reaction Questionnaire (TRQ) (Wilson et al., 1991). The Tinnitus Functional Index (TFI) (Meikle et al., 2012) was used in five studies. Regarding the psychoacoustic properties of tinnitus, we found that they were measured in ten studies but were rarely used in the analysis. Residual Inhibition (RI) was not measured. Details of the frequency of using each instrument are summarized in Table 2.

$<$ Table 2>

\section{Evidence of a relationship}

Evidence of a relationship between stress and tinnitus was collected in 26 studies. These studies reported association between the (a) onset of tinnitus and stress, (b) levels of stress and its effects in those with tinnitus, or (c) a statistical correlation between selfreported tinnitus and stress levels.

\section{a) Onset of tinnitus and stress}

Three studies reported the percentage of patients who associated the onset of their tinnitus with stress. These percentages were 13.5\% (n=200) (Y1ldırım et al., 2017), $14.2 \%(n=260)($ Muller et al., 2016), and 28.3\% $(n=350)$ (Probst et al., 2017). In Y1ldırım et al. (2017), patients clearly described the occurrence of at least one stressful event that induced or worsened their tinnitus.

\section{b) Stress levels and effects}

Tinnitus patients tended to report a moderate level of stress in their lives (Dineen et al., 1997). Patients with high self-reported tinnitus severity had significantly higher levels of stress as measured by PSQ vs TQ-52 (Olze et al., 2011) and MPS-9 (Psychological Stress Measurement) vs THI (Frachet et al., 2017). Higher levels of subjective stress 
were associated with louder and more distressing tinnitus (Probst et al., 2017). Tinnitus patients showed higher PSQ scores than healthy controls (Betz et al., 2017). Gomaa et al. (2014) stated that $73 \%$ of tinnitus patients, who also had hearing loss, suffered from stress. They described a correlation between the duration and severity of tinnitus and the severity of stress. In a group of workers at a call centre aged $22-45$ years, $33.1 \%$ reported tinnitus and $62.1 \%$ described high job-related stress level (Saruhanoglu et al., 2017).

\section{c) Stress-related symptoms in tinnitus}

Stress-related symptoms such as headache, tension in facial muscles and sleep disturbance were more frequent when the level of tinnitus was high. This is reported by Lindberg et al. (1984) who assess the grade of tinnitus by using Klockhoff and Lindblom grading system (1967). The authors concluded that the frequency of these symptoms strongly correlate with the grade of tinnitus. Similarly, Ciminelli et al. (2018) reported that $65 \%$ of participants described presence of stress symptoms (Lipp's Inventory for Symptoms of Stress (ISSL)), and this percentage is higher in patients who had higher scores on THI. On the Life Event Scale and the Daily Hassles Scale, patients with sudden sensory hearing loss and tinnitus had significantly higher scores than those in control group (Schmitt et al., 2000).

\section{d) The effect of stress on tinnitus perception}

The effect of stress on tinnitus was reported in six studies. 53.8\% of tinnitus patients reported that stress intensified their tinnitus (Muller et al., 2016). Hebert and Lupien (2009) described increase in the subjective stress and salivary cortisol after exposure to noise, accompanied by an increase in 'intensity' of tinnitus. Ecological Momentary Assessment (EMA) showed that tinnitus (bothersomeness and loudness) and stress changed together across time (Goldberg et al., 2017); the author concluded that 
temporary changes in bothersome tinnitus is strongly associated with levels of perceived stress. (Probst et al., 2016; Probst et al., 2017) also measured stress and tinnitus (loudness and distress) at different times of the day using $\mathrm{VAS}_{0-1}$, and reported that stress mediated the association between tinnitus loudness and tinnitus distress (Probst et al., 2016).

\section{e) Correlations between self-reported stress and tinnitus}

Positive correlation between self-reported tinnitus and stress measurements were reported in 15 studies (Muller et al., 2016; Bencsik et al., 2015; Ahmed et al., 2017; Panagiotopoulos et al., 2015; Park et al., 2017; Salviati et al., 2013; Brueggemann et al., 2019; Frachet et al., 2017; Han et al., 2019; Olze et al., 2011; Jackson, 2019; Gerber et al., 1985; Dineen et al., 1999). The strength of this correlation varied according to the instruments used from 0.26 (FTQ and PSQ-30) to 0.67 (THI and SVS) (Table 3).

$<$ Table 3>

Non-significant correlations were found between TRQ and DSP scores (Dineen et al., 1999), between the Stressful Life Events scores and tinnitus severity questionnaire scores (not specified) (Gerber et al., 1985), and between Tinnitus VAS $_{1-100}$ on one hand and Severity of Recent Life Event (SRLE) and PSS-10 on the other (Jackson, 2019).

\section{Biomarkers of stress in tinnitus}

\section{a) Cortisol}

Cortisol, either in saliva or blood, was the most commonly assessed biomarker ( $\mathrm{n}=6$ studies). Kim et al. (2013) reported that single-point blood cortisol levels did not correlate with the VAS, THI, or Korean-Brief Encounter Psychosocial Instrument (KBEPSI). Tinnitus participants who had high TRQ scores (>18) presented chronically above median salivary cortisol (CAMC) i.e. their cortisol were more frequently elevated 
than low distressed and healthy controls (Hebert et al., 2004). Salivary samples were taken five times across the day to assess CAMC. Cortisol Awakening Response (CAR) was assessed by measuring the Area Under The Curve with respect to increase (AUCi) (Jackson, 2019). Distressed tinnitus participants (TFI scores >25) had significantly lower AUCi than non-distressed tinnitus participants (TFI <25) and controls. Jackson 2019 also found that AUCi levels and tinnitus distress levels (VAS $\mathrm{VA-100}$ ) measured at awakening, after 6 hours and after 12 hours had moderate negative and statistically significant correlation. This indicates that AUCi levels may predict tinnitus distress levels later in the day.

Salivary cortisol, that measured at different points after exposure stress or noise, displayed a blunted and delayed response (Hebert and Lupien, 2007; Hebert and Lupien, 2009). Aydin and Searchfield (2019) did not found significant changes in cortisol and cortisone levels after exposure to BBN and Nature sound in tinnitus particiants. However, their finding was based on a single point measurement before and after exposure, and they reported higher variation in cortisol levels among the participants.

Tinnitus patients' response to a dexamethasone suppression test was stronger than healthy participants; the percentage reduction in salivary cortisol after dexamethasone was significantly higher than that of healthy controls despite similar basal cortisol levels, implying an increase in glucocorticoid sensitivity in tinnitus patients (Simoens and Hebert, 2012).

\section{b) Other biomarkers}

Stress-related immunological biomarkers (cytokines, Tissue Necrotic Factor, interleukins [IL-6, IL-10]) were compared in patients with chronic tinnitus and healthy 
participants; only levels of IL-6 were higher in tinnitus patients (Weber et al., 2002). Elevated NE or 5-HIAA were more frequently observed in tinnitus patients than controls, and ranged widely among patients, but differences were not statistically significant (Kim et al., 2013).

Indicators of sympathetic activity were assessed in six studies. (Betz et al., 2017) reported no difference between tinnitus and non-tinnitus group, and no relationship between heart rate variability and TQ scores. Ylikoski et al. (2017) reported decrease in heart rate variability after TRT (Aydin and Searchfield, 2019) reported decrease in Blood pressure after BBN and nature sound. Crocetti et al. (2018), Kirsch and Blanchard (1987), Weise et al. (2008) assessed muscle tension, skin conductance, and change in temperature and they did not report any significant findings.

\section{Change in tinnitus and stress after intervention}

Stress and tinnitus were assessed before and after tinnitus management in 22 studies. The reported changes were inconsistence as seen in Table 4.

\section{$<$ Table 4>}

Cognitive Behavioural Therapy (CBT) was used in four interventional studies. CBT was part of a multimodal treatment in Brüggemann et al. (2018) who reported improvement in TQ and PSQ after 7 days of multimodal treatment and this improvement was maintained in 5 years follow-up. In Hesser et al. (2012) THI and PSS scores improved after internet-delivered CBT and Acceptance and Commitment Therapy (ACT); there was a significant linear decrease in tinnitus distress and PSS scores. Li et al. (2019) introduced CBT with masking therapy and found that cortisol and tinnitus (THI) levels become lower after treatment, but interleukins (IL-2) level become significantly higher. In contrast, Abbott et al. (2009) found no significant 
changes in TRQ or DASS stress scores (both were low at baseline) after CBT.

Changes in the level of stress and tinnitus after relaxation therapy were reported in four studies. Kirsch and Blanchard (1987) used progressive relaxation training and biofeedback therapy with six patients. A reduction in tinnitus severity and stress level was reported but was non-concomitant. Another study showed a reduction in tinnitus and stress levels after weekly yoga sessions (Koksoy et al., 2017).

Tinnitus and stress levels decreased after progressive muscle relaxation (Weber et al., 2002); PSQ scores and Tissue Necrotic Factor alpha (TNF- $\alpha$ ) levels decreased significantly in tinnitus patients compared to non-tinnitus controls. Weise et al. (2008) measured tinnitus severity using the TQ, the electromyogram (EMG), and skin conductance before and after biofeedback management. No relationship analyses were reported, as the main aim of this study was to assess the test-retest reliability of EMG and skin conductance.

Sound-based management, which aims to mask or distract the patient from tinnitus, was the intervention of choice in eight studies. In four different records, Olze et al. (2012; 2011), Haussler et al. (2019) and Ketterer et al. (2018), the effect of cochlear implants (CI) on tinnitus and stress levels was assessed. Both decreased after CI when patients initially had high levels of tinnitus distress and stress (PSQ higher than 0.33). Ketterer et al. (2018) reported no change in PSQ after 6- and 12-month post-CI (initial PSQ scores were $0.3 \pm 18)$.

Durai and Searchfield (2017) used broadband noise and described a decrease in stress and tinnitus scores. On the other hand, no change occurred in stress level after musicbased treatment in Attanasio et al. (2012) but tinnitus intensity improved significantly. No changes occurred after broadband noise exposure in Dineen et al. (1999). 
Tinnitus Retraining Therapy (TRT) (Jastreboff and Hazell, 2008) decreased both tinnitus and stress levels. In Seydel et al. (2010), more improvement was noticed in participants with higher level of stress. The improvement in TQ and PSQ scores were maintained 5 years post-multimodal TRT (Brüggemann et al., 2018).

Two studies used a pharmacological intervention. Aoki et al. (2012) aimed to evaluate whether the use of lyophilised powder of enzymolysed honeybee larvae provides a therapeutic effect on tinnitus-related symptoms. They reported a decline in cortisol/dehydroepiandrosterone sulfate (DHEA) ratio after this treatment but tinnitus distress did not improve. Frachet et al. (2017) studied the effect of Auditism ${ }^{\circledR}$ (a combination of Ginkgo Biloba, melatonin, magnesium, zinc) which improved the level of tinnitus in $40.1 \%$ and level of stress in $21.8 \%$ of the participants. Ylikoski et al. (2017) reported an improvement in HRV after transcutaneous vagal nerve stimulation (tVNS), but they did not indicate if there was a change in tinnitus distress.

\section{Discussion}

The aim of this scoping review was to catalogue research to date evaluating the relationship between stress and tinnitus. We first reviewed measurements used in the included studies, which typically focused on one psychological concept of stress at a time, most often perceived stress. Psychoacoustic measures of tinnitus were rarely analysed in the included studies. None of these studies provided a clear rationale for not including these measures in the analysis, therefore no conclusion could be drawn about the effect of stress on the perceptual qualities of tinnitus. A number of tinnitus instruments were used in the included studies. For these instruments, there is varied evidence for the reliability and validity. For example, questions have been raised on the questionnaire structures, overlap of content and depth of information being measured 
(Tyler et al., 2007; Fackrell et al., 2016; Newman et al., 2014; Kamalski et al., 2010).

In this review, we asked whether exposure to stress plays a role in the pathophysiology of tinnitus. As the association between an outcome and an exposure is a first step toward defining a cause-effect relationship and it should be studied for consistency and specificity (Lucas and McMichael, 2005), we need to find whether stress acts as stimulus and causes tinnitus, and/or whether exposure to stress modulate tinnitus. The collated evidence showed that up to $29 \%$ of tinnitus patients associated the onset of tinnitus with stress, as per question number 7 in the Tinnitus Sample Case History questionnaire (Langguth et al., 2007). However, no guiding definition of stress is given in this question. Therefore, it is not clear if the tinnitus was associated with the occurrence of events or with the emotional distress that occurred when the event was perceived as stressful. As advised by Cohen et al. (1995), the best strategy to associate the onset of any disorder with stress is to assess it with a stimulus-based instruments. These instruments were used in just three studies (Gerber et al., 1985; Schmitt et al., 2000; Jackson, 2019), neither of which examined the onset of tinnitus with the occurrence of stressful event. Schmitt et al. (2000) reported higher Life Events Scale and Daily Hassles scores in those with sensorineural hearing loss and/or tinnitus than controls. But, they did not report the findings in the 'tinnitus only' group separately. Jackson (2019) reported negative, but non-significant, correlation with the severity of tinnitus.

It is obvious at this stage that not all tinnitus patients associated the onset of tinnitus with stress. This may be because the effect of daily hassles and recent life events on the onset of tinnitus was overlooked, or the inclusion of participants with long-term tinnitus resulting in a recall bias. Moreover, as tinnitus is a multifactorial and heterogeneous disorder with many different complaints associated with it, as previously reported 
(Sanchez and Stephens, 1997; Sanchez and Stephens, 2000; Tyler and Baker, 1983;

Watts et al., 2018), any study conducted in tinnitus is often rich in confounding factors. Similarly, most studies did not provide extensive detail about stress, e.g. whether it was associated with disease, or was acute or chronic. Therefore, this association will not simply imply a causal relationship between tinnitus and stress.

Several studies in this review showed that stress increased the bothersomeness and loudness of tinnitus. This is consistent with Baigi et al. (2011) who suggested that stress is a key factor in modulating the severity of tinnitus, in this study no instrument was used to assess the levels of stress and tinnitus. Studies included in this review used different methodological approach. In two included studies (Muller et al., 2016; Y1ldırım et al., 2017), participants were asked whether stress intensified their tinnitus, around half of the patients, answered the question positively. Other studies indicated a simultaneous fluctuation of tinnitus and stress levels based on daily monitoring. Their finding supports the notion that stress modulates tinnitus perception, but they also asked participants whether they felt stressed without providing a definition of stress. We observed that not all the participants associated tinnitus and stress, and there is variability in cortisol levels in tinnitus population. This may indicate a possibility to use stress levels to split tinnitus population into groups. Comparing these groups may reveal additional factors that likely mediate this relationship.

The biophysiological markers of stress are objective measures, and the measured response in tinnitus participants was different from non-tinnitus controls. Levels of IL-6 were higher in tinnitus patients than a younger control group (Weber et al., 2002). In general, IL-6 increases with age (Puzianowska-Kuźnicka et al., 2016; Hager et al., 1994) which may indicate that this finding is not specific to tinnitus. Salivary cortisol secretion in tinnitus patients exposed to short-term mental stress or noise was blunted 
and delayed in comparison to age-matched controls and was more so in those with higher tinnitus distress. This implies a dysregulation in the Hypothalamus-PituitaryAdrenal axis (Mazurek et al., 2015). This is a momentary measurement and the assessment of long-term cortisol levels will reveal the significance of this finding. Other sampling techniques like 24-hour urine collection, which provides cortisol level over an entire day (Dantzer et al., 2014), and hair analysis, which gives reliable retrospective cortisol levels up to 5 months (Stalder et al., 2017), are examples of sampling techniques to assess long-term cortisol secretion.

The indicators of the sympathetic-adrenal-medullary axis response (Skin conductance, EMG, and HRV) were also assessed. According to Crocetti et al. (2018), attention focus on tinnitus does not seem to produce sympathetic activation. Only HRV in tinnitus patients was different from healthy controls and improved after tVNS treatment in patients who had high levels originally (Ylikoski et al., 2017). But these findings are limited by the effect of confounders as HRV is not an exclusive biomarker of the autonomic nervous system and affected by many factors such as the age, physical activity, and blood pressure (Valentini and Parati, 2009). It is noteworthy that none of the previous studies examined changes in stress biomarkers in patients with recent onset tinnitus, at least as a separate group.

Many included studies reported a positive correlation between measurement of tinnitus and perceived stress measurements. However, this probably resulted from the overlap of psychological constructs of these questionnaires (Hall, 2017). This is noticeable by looking at the difference in the strength of the correlation (Table 2). A moderate correlation was found between perceived stress measurements and tinnitus measurements that mainly assess distress in tinnitus patients (THI, TFI, TQ, TRQ), and a weak correlation was between PSQ and both the FTQ and TCS which assess tinnitus- 
related fear. These studies used similar statistical testing, which opens the field for a systematic synthesis for the strength of this correlation.

Yet, self-reported levels of stress were higher in tinnitus patients than non-tinnitus controls (Betz et al., 2017). Patients with decompensated tinnitus had higher stress scores than patients with chronic pain (Zirke et al., 2013). Stress manifested with different symptoms. These symptoms occurred more frequently in those with tinnitus than those without, and were much more common when the subjective grading of tinnitus was higher. Likewise, van Dijk et al. (1987) reported an interrelation between tinnitus and the frequency of stress symptoms. These findings could be considered as evidence of a relationship. However, due to the potential overlap in contents between the questionnaires, these conclusions do need to be interpreted with caution. These findings and the similar statistical testing do however indicate the need for a systematic review to examine and synthesise the evidence across studies and for future studies to focus on comparing the level of perceived stress and stress symptoms in tinnitus patients with other patient populations e.g. patients with fibromyalgia). It is important to consider confounders and shared underlying variables that may affect the correlation between tinnitus and stress measurements. In addition, stress questionnaires are prone to different kinds of method bias which may affect the estimate of the relationship between two constructs (Podsakoff et al., 2012; Weckesser et al., 2019).

We also catalogued how tinnitus management affected the level of stress and tinnitus. The type of tinnitus management, whether it was stress- or sound-based management did not seem to influence how their levels changed. The most effective management of tinnitus has not been established and the efficacy of most tinnitus managements lacks supportive evidence (Makar et al., 2017; Hoare et al., 2011). It appears that changes after CBT (Hesser et al., 2012) and CI (Olze et al., 2011; Olze et al., 2012) were more 
likely to occur if the baseline level was high, and less likely to occur if the baseline level was low (Abbott et al., 2009). Thus, it is questionable if the baseline levels of tinnitus and stress have an influence on the outcome of intervention. This requires further investigation to estimate whether the selection of participants with high tinnitus level influences the outcome.

It was not possible to conduct a critical appraisal which is an optional step in scoping review methodology and not an essential aim (Munn et al., 2018; Tricco et al., 2018). Quality assessment does not form part of the scoping review which is a limitation of the method (Arksey and O'Malley, 2005). But this does not affect their ability to identify sources of the literature and makes it a robust method (Davis et al., 2009). We decided prospectively not to appraise studies as it was not relevant to the stated purpose of the review; to catalogue the evidence of the relationship. In addition, the included studies varied in design and outcome measures which would require a complex set of tools to conduct a meaningful appraisal. As such we also cannot in this review provide specific methodological recommendations considering tinnitus research; the reader is referred to general methodological considerations in Hall et al. (2018b) and Tyler et al. (2007).

\section{Recommendations and future directions}

This review can be used as guidance to conduct a systematic review of the use of specific stress instruments in this population or to design a study to assess the relationship under review. The suggestions and recommendations for future studies are:

1. To associate the onset of tinnitus with stress, it is important to use stimulusbased instruments or structured interviews, and study the association in recentonset tinnitus patients. 
2. We recommend the use of a guiding definition of stress to ensure participants and readers clearly understand the stress concept being investigated in the same manner.

3. When studying the changes in stress biomarkers in tinnitus patients, it is important to also consider psychological (depression and anxiety) and physical co-morbidities that may affect long-term and short-term stress biomarkers. Also, confounding factors, such as age, and hearing impairment, should be considered in the analysis.

4. It is important to compare stress-related biomarkers in age-matched groups, be measured at different points, and correlated with the changes in tinnitus severity.

5. We recommend to include the psychoacoustic measurements of tinnitus in the analysis if they were used, and to report the findings.

6. It should be acknowledged that psychometric tinnitus measurements assess the impact of tinnitus on various constructs or domains of quality of life (Kennedy et al., 2004). Therefore, the interpretation of the tinnitus severity should be based on the psychometric properties of the instruments they used.

There are opportunities to design studies and to conduct reviews that broaden our understanding of the role of stress in developing tinnitus. Many alternative methodological approaches should be considered. For example, studying more than one concept of stress using reliable measurements rather than focusing on one psychological concept of stress at a time. Daily monitoring and measuring of long-term self-reported stress and biomarkers levels may lead to significant findings as the stressful events that a person faces are varied in magnitude and quantity day-by-day. An additional consideration is to compare stress levels between different groups such as tinnitus and non-tinnitus groups, recent-onset and chronic tinnitus, or distresses and non-distressed 
tinnitus groups. This may reveal that not all tinnitus patients are vulnerable to stress or the existence of other confounding factors.

There are some reviewed studies that have fulfilled some of the abovementioned recommendations. For example, Jackson (2019) measured stress in its three different concepts. He used salivary cortisol as a biophysiological marker, SRLE as a stimulusbased instrument, and PSS-10 to measure the perceived stress. Based on the findings of this scoping review, we are also conducting a study in which we used DHS, RLCQ, DASS and hair cortisol (Elarbed et al., 2019). We also measured tinnitus loudness psychoacoustically for inclusion in the final analysis. With the existence of a wide variety of stress and tinnitus instruments, that future research uses them widely and simultaneously.

\section{Limitation}

The inclusion criteria of this review was limited to studies published in English only and relevant studies published in non-English languages would have been missed. However, the included studies were conducted in English and non-English speaking countries. We put a limitation on the age of participants and this led to the exclusion of 13 studies where at least some adults were included. For a systematic review, we recommend that data should be included by separating out the adult from child data. That was beyond the aim of this scoping review. Lastly, we included studies that measured stress and tinnitus separately. This lead to exclude studies that assessed stress in tinnitus population but did not assess the level of tinnitus.

\section{Conclusion}

The inconclusive results is enhancing understanding of the relationship, as the evidence suggests as it stands that the relationship between stress and tinnitus could go either way and that more research is needed on investigating the relationship, which 
recommendations are provided from the review as to the gaps in research. Several questions remain to be answered, such as the direction of this relationship, the effect of stress on the characteristics of tinnitus, and the role of stress mediators in tinnitus physiopathology. There is considerable room for improvement and for conducting further research about this relationship. This review highlighted some shortcomings and possible solutions that can be used as a reference point when designing studies investigating the relationship between stress and tinnitus.

\section{Conflicts of interest}

No conflict is declared.

\section{Acknowledgement}

$\mathrm{AE}$ is a PhD student funded by a scholarship from Libyan government. This review presents independent research supported by the National Institute for Health research (NIHR). The views expressed in this article are those of the authors and not necessarily those of the NHS, the NIHR, or the Department of Health and Social Care. 


\section{Reference}

Abbott JA, Kaldo V, Klein B, et al. (2009) A cluster randomised trial of an internetbased intervention program for tinnitus distress in an industrial setting. Cognitive Behaviour Therapy 38: 162-173. https://doi.org/10.1080/16506070902763174

Ahmed B, Ahmed A, Aqeel M, et al. (2017) Impact of tinnitus perception on psychological distress in male and female tinnitus patients. Foundation University Journal of Psychology 1: 1-26.

Andersson G and McKenna L. (2006) The role of cognition in tinnitus. Acta OtoLaryngologica 126: 39-43. 10.1080/03655230600895226

Aoki M, Wakaoka Y, Hayashi H, et al. (2012) Effect of Lyophilized Powder Made From Enzymolyzed Honeybee Larvae on Tinnitus-Related Symptoms, Hearing Levels, and Hypothalamus-Pituitary-Adrenal Axis-Related Hormones. Ear Hear 33: 430-436. 10.1097/AUD.0b013e318234f024

Arksey H and O'Malley L. (2005) Scoping studies: towards a methodological framework. International Journal of Social Research Methodology 8: 19-32. https://doi.org/10.1080/1364557032000119616

Attanasio G, Cartocci G, Covelli E, et al. (2012) The Mozart effect in patients suffering from tinnitus. Acta Oto-Laryngologica 132: 1172-1177. https://doi.org/10.3109/00016489.2012.684398

Aydin N and Searchfield GD. (2019) Changes in tinnitus and physiological biomarkers of stress in response to short-term broadband noise and sounds of nature. Complementary Therapies in Medicine 46: 62-68. https://doi.org/10.1016/j.ctim.2019.07.018

Baguley D, McFerran D and Hall D. (2013) Tinnitus. Lancet 382: 1600-1607. https://doi.org/10.1016/s0140-6736(13)60142-7

Baigi A, Oden A, Almlid-Larsen V, et al. (2011) Tinnitus in the general population with a focus on noise and stress: a public health study. Ear and hearing 32: 787-789. https://doi.org/10.1097/AUD.0b013e31822229bd

Bencsik B, Tamas L, Trimmel K, et al. (2015) Hungarian adaptation of the Tinnitus Handicap Inventory: reliability and validity. European archives of oto-rhinolaryngology 272: 2243-2248. https://doi.org/10.1007/s00405-014-3138-8

Betz LT, Muhlberger A, Langguth B, et al. (2017) Stress Reactivity in Chronic Tinnitus. Scientific Reports 7: 1-9. https://doi.org/10.1038/srep41521

Boals A and Banks JB. (2012) Effects of traumatic stress and perceived stress on everyday cognitive functioning. Cognition and Emotion 26: 1335-1343. 10.1080/02699931.2011.651100

Brueggemann P, Seydel C, Schaefer C, et al. (2019) ICD-10 Symptom Rating questionnaire for assessment of psychological comorbidities in patients with chronic tinnitus. HNO: 1-5. 10.1007/s00106-019-0625-7

Brüggemann P, Otto J, Lorenz N, et al. (2018) Long-term changes in multimodal intensive tinnitus therapy: A 5-year follow-up. HNO 66: 34-38. 10.1007/s00106-0170463-4 
Cederroth CR, Gallus S, Hall DA, et al. (2019) Towards an Understanding of Tinnitus Heterogeneity. Frontiers in aging neuroscience 11: 53.

Ciminelli P, Machado S, Palmeira M, et al. (2018) Tinnitus: The sound of stress? Clinical Practice and Epidemiology in Mental Health 14: 264-269. http://dx.doi.org/10.2174/1745017901814010264

Clarke NA, Henshaw H, Akeroyd MA, et al. (2020) Associations Between Subjective Tinnitus and Cognitive Performance: Systematic Review and Meta-Analyses. Trends in Hearing 24: $2331216520918416.10 .1177 / 2331216520918416$

Cohen S, Kamarck T and Mermelstein R. (1983) A global measure of perceived stress. Journal of Health and Social Behavior 24: 385-396.

Cohen S, Kessler RC and Gordon LU. (1995) Strategies for measuring stress in studies of psychiatric and physical disorders. In: S. Cohen, Kessler RC and Gordon LU (eds) Measuring stress: A guide for health and social scientists. New York, NY, US: Oxford University Press, 3-26.

Crocetti A, Ambrosetti U and Del Bo L. (2018) Flight or fight tinnitus? Tinnitus coping over time and psychophysiological reactions. Activitas Nervosa Superior Rediviva 60: 49-54.

Crocetti A, Forti S, Ambrosetti U, et al. (2009) Questionnaires to evaluate anxiety and depressive levels in tinnitus patients. Otolaryngology-head and neck surgery 140: 403-405. https://doi.org/10.1016/j.otohns.2008.11.036

Dantzer B, Fletcher QE, Boonstra R, et al. (2014) Measures of physiological stress: a transparent or opaque window into the status, management and conservation of species? Conservation Physiology 2: cou023-cou023. https://doi.org/10.1093/conphys/cou023

Davis K, Drey N and Gould D. (2009) What are scoping studies? A review of the nursing literature. International journal of nursing studies 46: 1386-1400.

Dineen R, Doyle J and Bench J. (1997) Audiological and psychological characteristics of a group of tinnitus sufferers, prior to tinnitus management training. British journal of audiology 31: 27-38. https://doi.org/10.3109/03005364000000006

Dineen R, Doyle J, Bench J, et al. (1999) The influence of training on tinnitus perception: an evaluation 12 months after tinnitus management training. British journal of audiology 33: 29-51.

Durai M and Searchfield G. (2016) Anxiety and depression, personality traits relevant to tinnitus: A scoping review. International Journal of Audiology 55: 605-615. http://dx.doi.org/10.1080/14992027.2016.1198966

Durai M and Searchfield GD. (2017) A mixed-methods trial of broad band noise and nature sounds for tinnitus therapy: Group and individual responses modeled under the adaptation level theory of tinnitus. Frontiers in aging neuroscience 9: 1-22.

Duric V, Clayton S, Leong ML, et al. (2016) Comorbidity Factors and Brain Mechanisms Linking Chronic Stress and Systemic Illness. Neural Plasticity 2016: 116. https://doi.org/10.1155/2016/5460732

Fackrell K, Hall DA, Barry JG, et al. (2016) Psychometric properties of the Tinnitus Functional Index (TFI): Assessment in a UK research volunteer population. Hear Res 335: 220-235. 10.1016/j.heares.2015.09.009 
Fisher GG, Chaffee DS, Tetrick LE, et al. (2017) Cognitive Functioning, Aging, and Work: A Review and Recommendations for Research and Practice. Journal of Occupational Health Psychology 22: 314-336. 10.1037/ocp0000086

Frachet B, Portmann D and Allaert FA. (2017) Observational study to assess the effect of Audistim(r) on the quality of life of patients presenting with chronic tinnitus. Revue de Laryngologie Otologie Rhinologie 138: 79-85.

Gerber KE, Nehemkis AM, Charter RA, et al. (1985) Is tinnitus a psychological disorder? International journal of psychiatry in medicine 15: 81-87.

Goldberg RL, Piccirillo ML, Nicklaus J, et al. (2017) Evaluation of Ecological Momentary Assessment for Tinnitus Severity. JAMA Otolaryngol Head \& Neck Surgery 143: 700-706. https://doi.org/10.1001/jamaoto.2017.0020

Gomaa MA, Elmagd MH, Elbadry MM, et al. (2014) Depression, Anxiety and Stress Scale in patients with tinnitus and hearing loss. European archives of oto-rhinolaryngology 271: 2177-2184. https://doi.org/10.1007/s00405-013-2715-6

Hager K, Machein U, Krieger S, et al. (1994) Interleukin-6 and selected plasma proteins in healthy persons of different ages. Neurobiology of Aging 15: 771-772. https://doi.org/10.1016/0197-4580(94)90066-3

Hall DA. (2017) Outcome measures associated with perceived stress. Tinnitus and Stress. Springer, 173-200.

Hall DA, Fackrell K, Li AB, et al. (2018a) A narrative synthesis of research evidence for tinnitus-related complaints as reported by patients and their significant others. Health and quality of life outcomes 16: 61.

Hall DA, Smith H, Hibbert A, et al. (2018b) The COMiT'ID Study: Developing Core Outcome Domains Sets for Clinical Trials of Sound-, Psychology-, and Pharmacology-Based Interventions for Chronic Subjective Tinnitus in Adults. Trends in Hearing 22: 2331216518814384-2331216518814384. $10.1177 / 2331216518814384$

Hallam RS, Jakes SC and Hinchcliffe R. (1988) Cognitive variables in tinnitus annoyance. The British journal of clinical psychology 27: 213-222.

Han TS, Jeong JE, Park SN, et al. (2019) Gender differences affecting psychiatric distress and tinnitus severity. Clinical Psychopharmacology and Neuroscience 17: 113-120. http://dx.doi.org/10.9758/cpn.2019.17.1.113

Haussler SM, Kopke V, Knopke S, et al. (2019) Multifactorial positive influence of cochlear implantation on patients with single-sided deafness. Laryngoscope. 10.1002/lary.28007

Hebert S and Lupien SJ. (2007) The sound of stress: blunted cortisol reactivity to psychosocial stress in tinnitus sufferers. Neuroscience letters 411: 138-142. https://doi.org/10.1016/j.neulet.2006.10.028

Hebert S and Lupien SJ. (2009) Salivary cortisol levels, subjective stress, and tinnitus intensity in tinnitus sufferers during noise exposure in the laboratory. International journal of hygiene and environmental health 212: 37-44. https://doi.org/10.1016/j.ijheh.2007.11.005 
Hebert S, Paiement P and Lupien SJ. (2004) A physiological correlate for the intolerance to both internal and external sounds. Hearing Research 190: 1-9. https://doi.org/10.1016/s0378-5955(04)00021-8

Henry JA, Piskosz M, Norena A, et al. (2019) Audiologists and Tinnitus. American journal of audiology 28: 1059-1064.

Henry JA, Roberts LE, Caspary DM, et al. (2014) Underlying Mechanisms of Tinnitus: Review and Clinical Implications. Journal of the American Academy of Audiology 25: 5-126. https://doi.org/10.3766/jaaa.25.1.2

Hesser H, Gustafsson T, Lunden C, et al. (2012) A randomized controlled trial of internet-delivered cognitive behavior therapy and acceptance and commitment therapy in the treatment of tinnitus. Journal of Consulting and Clinical Psychology 80: 649-661. https://doi.org/10.1037/a0027021

Hoare DJ, Kowalkowski VL, Kang S, et al. (2011) Systematic review and metaanalyses of randomized controlled trials examining tinnitus management. The Laryngoscope 121: 1555-1564. https://doi.org/10.1002/lary.21825

Holmes TH and Rahe RH. (1967) The social readjustment rating scale. Journal of psychosomatic research 11: 213-218.

Jackson JG. (2019) The Cortisol Awakening Response: A Feasibility Study Investigating the Use of the Area Under the Curve With Respect to Increase as an Effective Objective Measure of Tinnitus Distress. American journal of audiology 28: 583-596.

Jastreboff PJ and Hazell JW. (2008) Tinnitus retraining therapy: Implementing the neurophysiological model: Cambridge University Press.

Kamalski DM, Hoekstra CE, van Zanten BG, et al. (2010) Measuring disease-specific health-related quality of life to evaluate treatment outcomes in tinnitus patients: a systematic review. Otolaryngol Head Neck Surg 143: 181-185. 10.1016/j.otohns.2010.03.026

Kanner AD, Coyne JC, Schaefer C, et al. (1981) Comparison of two modes of stress measurement: Daily hassles and uplifts versus major life events. Journal of behavioral medicine 4: 1-39.

Kennedy V, Wilson C and Stephens D. (2004) Quality of life and tinnitus. Audiological medicine 2: 29-40.

Ketterer MC, Knopke S, Hausler SM, et al. (2018) Asymmetric hearing loss and the benefit of cochlear implantation regarding speech perception, tinnitus burden and psychological comorbidities: a prospective follow-up study. European Archives of Oto-Rhino-Laryngology 275: 2683-2693. https://dx.doi.org/10.1007/s00405-018$\underline{5135-9}$

Kim DK, Chung DY, Bae SC, et al. (2013) Diagnostic value and clinical significance of stress hormones in patients with tinnitus. European Archives of Oto-RhinoLaryngology: 1-7. https://doi.org/10.1007/s00405-013-2785-5

Kirsch CA and Blanchard EB. (1987) A multiple-baseline evaluation of the treatment of subjective tinnitus with relaxation training and biofeedback. Biofeedback and SelfRegulation 12: 295-312. https://doi.org/10.1007/BF00998721 
Klockhoff I and Lindblom U. (1967) Meniére's Disease and Hydrochlorothiazide (Dichlotride®) - A Critical Analysis of Symptoms and Therapeutic Effects. Acta Oto-Laryngologica 63: 347-365. https://doi.org/10.3109/00016486709128769

Koksoy S, Eti CM, Karatas M, et al. (2017) The Effects of Yoga in Patients Suffering from Subjective Tinnitus. International Archives of Otorhinolaryngology: 9-13. https://doi.org/10.1055/s-0037-1601415

Kreuzer PM, Vielsmeier V and Langguth B. (2013) Chronic Tinnitus: an Interdisciplinary Challenge. Deutsches Ärzteblatt International 110: 278-284. https://doi.org/10.3238/arztebl.2013.0278

Langguth B, Goodey R, Azevedo A, et al. (2007) Consensus for tinnitus patient assessment and treatment outcome measurement: Tinnitus Research Initiative meeting, Regensburg, July 2006. In: Langguth B, Hajak G, Kleinjung T, et al. (eds) Progress in Brain Research. Elsevier, 525-536. https://doi.org/10.1016/S0079$\underline{6123(07) 66050-6}$

Lazarus RS and Folkman S. (1984) Stress, appraisal, and coping New York: New York : Springer.

Levac D, Colquhoun H and O'Brien KK. (2010) Scoping studies: advancing the methodology. Implementation Science 5: 69-69. https://doi.org/10.1186/1748-5908$\underline{5-69}$

Levenstein S, Prantera C, Varvo V, et al. (1993) Development of the Perceived Stress Questionnaire - A new tool for psychosomatic research. Journal of psychosomatic research 37: 19-32.

Li J, Jin J, Xi S, et al. (2019) Clinical efficacy of cognitive behavioral therapy for chronic subjective tinnitus. American Journal of Otolaryngology - Head and Neck Medicine and Surgery 40: 253-256. http://dx.doi.org/10.1016/j.amjoto.2018.10.017

Lindberg p, Lyttkens L, Melin L, et al. (1984) Tinnitus-incidence and handicap. Scandinavian Audiology 13: 287-291. https://doi.org/10.3109/01050398409042138

Lovibond PF and Lovibond SH. (1995) The structure of negative emotional states: comparison of the Depression Anxiety Stress Scales (DASS) with the Beck Depression and Anxiety Inventories. Behaviour research and therapy 33: 335-343.

Lucas RM and McMichael AJ. (2005) Association or causation: evaluating links between "environment and disease". Bulletin of the World Health Organization 83: 792-795.

Makar SK, Mukundan G and Gore G. (2017) Treatment of tinnitus: a scoping review. The international tinnitus journal 21: 144-156.

Mazurek B, Szczepek AJ and Hebert S. (2015) Stress and tinnitus. Hno 63: 258-265. https://doi.org/10.1007/s00106-014-2973-7

McEwen BS. (2005) Stressed or stressed out: What is the difference? Journal of Psychiatry and Neuroscience 30: 315-318.

Meikle MB, Henry JA, Griest SE, et al. (2012) The tinnitus functional index: development of a new clinical measure for chronic, intrusive tinnitus. Ear and hearing 33: 153-176. https://doi.org/10.1097/AUD.0b013e31822f67c0 
Milerova J, Anders M, Dvorak T, et al. (2013) The influence of psychological factors on tinnitus severity. General hospital psychiatry 35: 412-416. https://doi.org/10.1016/j.genhosppsych.2013.02.008

Mohamad N, Hoare DJ and Hall DA. (2016) The consequences of tinnitus and tinnitus severity on cognition: A review of the behavioural evidence. Hearing Research 332: 199-209.

Møller AR. (2016) Sensorineural tinnitus: its pathology and probable therapies. International journal of otolaryngology 2016: 2830157. 10.1155/2016/2830157

Monroe SM. (2008) Modern approaches to conceptualizing and measuring human life stress. Annu. Rev. Clin. Psychol. 4: 33-52.

Muller K, Edvall NK, Idrizbegovic E, et al. (2016) Validation of online versions of tinnitus questionnaires translated into Swedish. Frontiers in aging neuroscience 8: 115 .

Munn Z, Peters MDJ, Stern C, et al. (2018) Systematic review or scoping review? Guidance for authors when choosing between a systematic or scoping review approach. BMC Medical Research Methodology 18: 143. 10.1186/s12874-018-0611$\mathrm{x}$

Newman CW, Jacobson GP and Spitzer JB. (1996) Development of the tinnitus handicap inventory. Archives of Otolaryngology-Head \& Neck Surgery 122: 143148. https://doi.org/10.1001/archotol.1996.01890140029007

Newman CW, Sandridge SA and Jacobson GP. (2014) Assessing outcomes of tinnitus intervention. J Am Acad Audiol 25: 76-105. 10.3766/jaaa.25.1.6

Olze H, Grabel S, Forster U, et al. (2012) Elderly patients benefit from cochlear implantation regarding auditory rehabilitation, quality of life, tinnitus, and stress. The Laryngoscope 122: 196-203. https://doi.org/10.1002/lary.22356

Olze H, Szczepek AJ, Haupt H, et al. (2011) The impact of cochlear implantation on tinnitus, stress and quality of life in postlingually deafened patients. Audiology and Neurotology 17: 2-11. https://doi.org/10.1159/000323847

Panagiotopoulos G, Galanakis M, Varvogli L, et al. (2015) Validation of the Greek version of Mini Tinnitus Questionnaire as a Brief Screening Test for Assessment of Tinnitus-related Distress: our experience in 301 adult patients. Clinical otolaryngology 40: 363-369. https://doi.org/10.1111/coa.12383

Papathanasiou IV, Tsaras K, Neroliatsiou A, et al. (2015) Stress: Concepts, theoretical models and nursing interventions. American Journal of Nursing Science 4: 45-50.

Park SY, Han JJ, Hwang JH, et al. (2017) Comparison of tinnitus and psychological aspects between the younger and older adult patients with tinnitus. Auris Nasus Larynx 44: 147-151. https://doi.org/10.1016/j.an1.2016.04.007

Pattyn T, Van Den Eede F, Vanneste S, et al. (2016) Tinnitus and anxiety disorders: A review. Hearing Research 333: 255-265.

https://doi.org/10.1016/j.heares.2015.08.014

Peters MDJ, Godfrey CM, Khalil H, et al. (2015) Guidance for conducting systematic scoping reviews. International Journal of Evidence-Based Healthcare 13: 141-146. 10.1097/xeb.0000000000000050 
Pinto PCL, Marcelos CM, Mezzasalma MA, et al. (2014) Tinnitus and its association with psychiatric disorders: systematic review. The Journal of Laryngology \& Otology 128: 660-664. https://doi.org/10.1017/S0022215114001030

Podsakoff PM, MacKenzie SB and Podsakoff NP. (2012) Sources of method bias in social science research and recommendations on how to control it. Annual review of psychology 63: 539-569.

Probst T, Pryss R, Langguth B, et al. (2016) Emotional states as mediators between tinnitus loudness and tinnitus distress in daily life: Results from the "TrackYourTinnitus" application. Scientific Reports 6: 20382. https://doi.org/10.1038/srep20382

Probst T, Pryss RC, Langguth B, et al. (2017) Does tinnitus depend on time-of-day? An ecological momentary assessment study with the "trackyourtinnitus" application. Frontiers in aging neuroscience 9. https://doi.org/10.3389/fnagi.2017.00253

Puzianowska-Kuźnicka M, Owczarz M, Wieczorowska-Tobis K, et al. (2016) Interleukin-6 and C-reactive protein, successful aging, and mortality: the PolSenior study. Immunity \& Ageing : I \& A 13: 21. https://doi.org/10.1186/s12979-016-0076-x

Rajmohan V and Mohandas E. (2007) The limbic system. Indian journal of psychiatry 49: 132.

Rauschecker JP, Leaver AM and Mühlau M. (2010) Tuning out the noise: Limbicauditory interactions in tinnitus. Neuron 66: 819-826. https://doi.org/10.1016/j.neuron.2010.04.032

Rice VH. (2012) Handbook of stress, coping, and health : implications for nursing research, theory, and practice. , Thousand Oaks, Calif.: Thousand Oaks, Calif. : SAGE Publications.

Salviati M, Macri F, Terlizzi S, et al. (2013) The Tinnitus Handicap Inventory (THI) as a screening test for psychiatric comorbidity in patients with tinnitus. Psychosomatics: Journal of Consultation and Liaison Psychiatry 54: 248-256. https://doi.org/10.1016/j.psym.2012.05.007

Sanchez L and Stephens D. (1997) A tinnitus problem questionnaire in a clinic population. Ear Hear 18: 210-217. 10.1097/00003446-199706000-00004

Sanchez L and Stephens S. (2000) Perceived problems of tinnitus clinic clients at longterm follow up. Journal of Audiological Medicine 9: 94-103.

Saruhanoglu A, Gokcen-Rohlig B, Saruhanoglu C, et al. (2017) Frequency of temporomandibular disorder signs and symptoms among call center employees. Cranio-the Journal of Craniomandibular Practice 35: 244-249. https://doi.org/10.1080/08869634.2016.1216823

Saxena RP. (1980) Hans Selye, text book of stress, Laindon, Essex: Laindon, Essex : Journal of Chronic Diseases and Therapeutics Research.

Schmitt C, Patak M and Kroner-Herwig B. (2000) Stress and the onset of sudden hearing loss and tinnitus. The international tinnitus journal 6: 41-49.

Schneiderman N, Ironson G and Siegel SD. (2005) Stress and Health: Psychological, Behavioral, and Biological Determinants. Annual review of clinical psychology 1: 607-628. https://doi.org/10.1146/annurev.clinpsy.1.102803.144141 
Seydel C, Haupt H, Szczepek AJ, et al. (2010) Long-term improvement in tinnitus after modified tinnitus retraining therapy enhanced by a variety of psychological approaches. Audiology \& neuro-otology 15: 69-80. https://doi.org/10.1159/000231632

Simoens VL and Hebert S. (2012) Cortisol suppression and hearing thresholds in tinnitus after low-dose dexamethasone challenge. BMC Ear, Nose and Throat Disorders 12: 4. https://doi.org/10.1186/1472-6815-12-4

Stalder T, Steudte-Schmiedgen S, Alexander N, et al. (2017) Stress-related and basic determinants of hair cortisol in humans: A meta-analysis. Psychoneuroendocrinology 77: 261-274. https://doi.org/10.1016/j.psyneuen.2016.12.017

Tegg-Quinn S, Bennett RJ, Eikelboom RH, et al. (2016) The impact of tinnitus upon cognition in adults: A systematic review. International journal of audiology 55: 533540. 10.1080/14992027.2016.1185168

Tricco AC, Lillie E, Zarin W, et al. (2018) PRISMA Extension for Scoping Reviews (PRISMA-ScR): Checklist and Explanation. Ann Intern Med 169: 467-473. $10.7326 / \mathrm{m} 18-0850$

Tyler RS and Baker LJ. (1983) Difficulties experienced by tinnitus sufferers. Journal of Speech and Hearing disorders 48: 150-154.

Tyler RS, Oleson J, Noble W, et al. (2007) Clinical trials for tinnitus: study populations, designs, measurement variables, and data analysis. Prog Brain Res 166: 499-509. 10.1016/s0079-6123(07)66048-8

Valentini M and Parati G. (2009) Variables Influencing Heart Rate. Progress in Cardiovascular Diseases 52: 11-19. https://doi.org/10.1016/j.pcad.2009.05.004

van Dijk FJH, Souman AM and de Vries FF. (1987) Non-auditory effects of noise in industry - VI. A final field study in industry. International Archives of Occupational and Environmental Health 59: 133-145. https://doi.org/10.1007/BF00378491

Watts EJ, Fackrell K, Smith S, et al. (2018) Why Is Tinnitus a Problem? A Qualitative Analysis of Problems Reported by Tinnitus Patients. Trends Hear 22: 2331216518812250. 10.1177/2331216518812250

Weber C, Arck P, Mazurek B, et al. (2002) Impact of a relaxation training on psychometric and immunologic parameters in tinnitus sufferers. Journal of psychosomatic research 52: 29-33.

Weckesser LJ, Dietz F, Schmidt K, et al. (2019) The psychometric properties and temporal dynamics of subjective stress, retrospectively assessed by different informants and questionnaires, and hair cortisol concentrations. Scientific Reports 9: 1098. 10.1038/s41598-018-37526-2

Weise C, Heinecke K and Rief W. (2008) Stability of physiological variables in chronic tinnitus sufferers. Applied psychophysiology and biofeedback 33: 149-159. https://doi.org/10.1007/s10484-008-9058-x

Wilson PH, Henry J, Bowen M, et al. (1991) Tinnitus reaction questionnaire: psychometric properties of a measure of distress associated with tinnitus. Journal of speech and hearing research 34: 197-201.

Yildırım N, Aksoy S and Erdoğan O. (2017) Role of stressful life events as the precipitating factor for chronic tinnitus. Journal of Hearing Science 7: 138-138. 
Ylikoski J, Lehtimaki J, Pirvola U, et al. (2017) Non-invasive vagus nerve stimulation reduces sympathetic preponderance in patients with tinnitus. Acta Oto-Laryngologica 137: 426-431. http://dx.doi.org/10.1080/00016489.2016.1269197

Zirke N, Seydel C, Szczepek AJ, et al. (2013) Psychological comorbidity in patients with chronic tinnitus: analysis and comparison with chronic pain, asthma or atopic dermatitis patients. Quality of life research 22: 263-272.

https://doi.org/10.1007/s11136-012-0156-0 


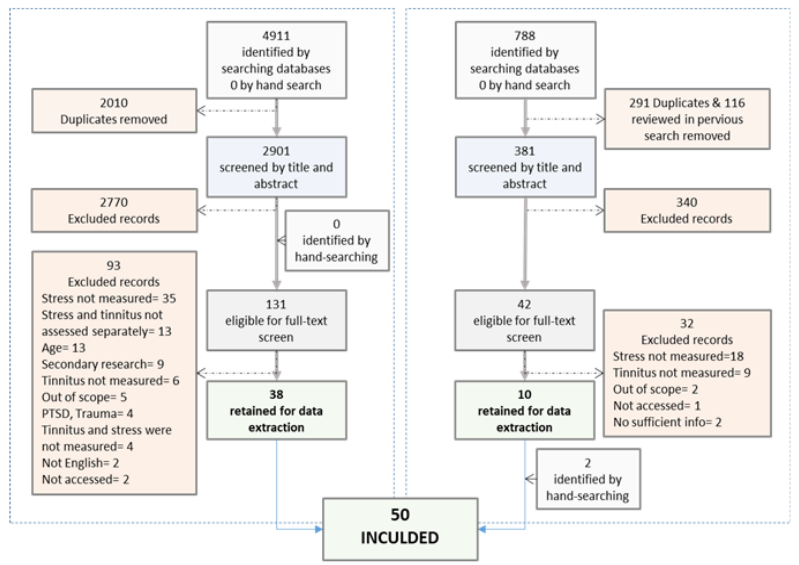

Fig. 1. Flow diagram details search process and count of included and excluded records of each stage of the review. We repeated the same steps in the updated search in July. 
Table 1: Search strategies examples

\begin{tabular}{ll} 
Database & Search term \\
\hline Pubmed & Tinnitus AND stress* AND adult AND human \\
PsycholNFO & tinnitus.mp. or exp tinnitus/ \\
& stress*.mp. or exp psychological stress/or exp \\
& stress/ \\
& 1 and 2 \\
& limit 3 to (human and English language) \\
\hline
\end{tabular}


Table 2: List of measurements used in the included studies.

\begin{tabular}{|c|c|c|}
\hline Perceived Stress Measurements & & frequency of using \\
\hline Perceived Stress Questionnaire & PSS & 11 \\
\hline Visual analogue Scale & VAS & 6 \\
\hline Depression Anxiety Stress Scale & DASS & 5 \\
\hline Perceived Stress Scale & PSS & 4 \\
\hline Korean-Brief Encounter Psychosocial Instrument & K-BEPSI & 3 \\
\hline Derogatis Stress Profile & DSP & 2 \\
\hline Psychological Stress Measurement & MSP & 2 \\
\hline \multicolumn{3}{|l|}{ Stimulus-based Stress Measurements } \\
\hline Recent Life Changes & RLC & 2 \\
\hline Severity of Recent Life Event & SRLE & 1 \\
\hline Daily Hassles Scale & DHS & 1 \\
\hline \multicolumn{3}{|l|}{ Symptoms of Stress Measurements } \\
\hline Stress Vulnerability Scale & VRS & 1 \\
\hline Lipp's Inventory for Stress Symptoms & ISSL & 1 \\
\hline Stress Symptoms Scale & SSS & 2 \\
\hline \multicolumn{3}{|l|}{ Stress Biophysiological Measurements } \\
\hline Cortisol & CORT & 9 \\
\hline Cortisone & CORTn & 1 \\
\hline dehydroepiandrosterone Sulphate & DHEAS & 1 \\
\hline Interleukins $(2,6,10)$ & IL & 3 \\
\hline Epinephrine \& norepinephrine & NE, EPI & 1 \\
\hline Tissue Necrotic Factor a & TNFa & 1 \\
\hline Blood Pressure & $\mathrm{BP}$ & 1 \\
\hline Heart Rate \& Heart Rate variability & HR, HRV & 4 \\
\hline Skin Conductance & SkD & 2 \\
\hline Electromyogram (Muscle contraction) & EMG & 3 \\
\hline Temperature & Temp & 3 \\
\hline \multicolumn{3}{|l|}{ Tinnitus Psychometric Measurements } \\
\hline Tinnitus Handicap Inventory & THI & 17 \\
\hline Tinnitus Questionnaire & TQ & 13 \\
\hline Tinnitus Reaction Questionnaire & TRQ & 7 \\
\hline Visual analogue Scale & VAS & 6 \\
\hline Tinnitus Functional Index & TFI & 5 \\
\hline Fear of Tinnitus Questionnaire & FTQ & 1 \\
\hline Tinnitus Catastrophic Scale & TCS & 1 \\
\hline Tinnitus Handicap Questionnaire & THQ & 1 \\
\hline Global Rating Bother Scale & GBS & 1 \\
\hline Tinnitus Severity Questionnaire & TSQ & 1 \\
\hline Tinnitus Acceptance Questionnaire & TAQ & 1 \\
\hline Tinnitus Fragebogen & $\mathrm{TF}$ & 1 \\
\hline \multicolumn{3}{|l|}{ Tinnitus Psychoacoustic Measurements } \\
\hline Pitch Matching & PM & 8 \\
\hline Loudness Matching & $\mathrm{LM}$ & 6 \\
\hline Minimum Masking Level & MML & 5 \\
\hline
\end{tabular}


Table 3: Correlation between tinnitus and stress subjective measurements.

\begin{tabular}{|c|c|c|c|c|c|}
\hline Author & $\begin{array}{l}\text { Tinnitus } \\
\text { measurements }\end{array}$ & $\begin{array}{l}\text { Stress } \\
\text { measurements }\end{array}$ & $\begin{array}{l}\text { Statistical } \\
\text { analysis }\end{array}$ & $\mathbf{r}$ & $\mathbf{p}$ \\
\hline \multirow[t]{2}{*}{ Ahmed et al. (2017) } & THI (male) & DASS (male) & NR & 0.54 & $<0.001$ \\
\hline & THI (female) & DASS (female) & NR & 0.53 & $<0.001$ \\
\hline Bencsik et al. (2015) & THI & PSS-4 & $\mathrm{PC}$ & 0.55 & $<0.01$ \\
\hline Betz et al. (20017) & TQ & PSQ & $\mathrm{PC}$ & 0.62 & $=0.005$ \\
\hline Brueggemann et al (2019) & TQ & PSQ & $\mathrm{PC}$ & 0.53 & $<0.01$ \\
\hline Frachet et al. (2017) & THI & MPS-9 & $\mathrm{PC}$ & 0.67 & $<0.000$ \\
\hline \multirow[t]{2}{*}{ Han et al. (2019) } & THI (male) & K-BEPSI (male) & $\mathrm{PC}$ & 0.59 & $<0.01$ \\
\hline & THI (female) & K-BEPSI (female) & $\mathrm{PC}$ & 0.29 & ns \\
\hline \multirow[t]{4}{*}{ Muller et al. (2016) } & THI & PSQ-30 & $\mathrm{SC}$ & 0.47 & $<0.01$ \\
\hline & TFI & PSQ-30 & $\mathrm{SC}$ & 0.41 & $<0.01$ \\
\hline & TCS & PSQ-30 & $\mathrm{SC}$ & 0.34 & $<0.01$ \\
\hline & FTQ & PSQ-30 & $\mathrm{SC}$ & 0.26 & $<0.01$ \\
\hline Olze et al. (2011a) & TQ & PSQ & $\mathrm{SC}$ & 0.58 & $<0.001$ \\
\hline Olze et al. (2011b) & TQ & PSQ & $\mathrm{SC}$ & 0.44 & $<0.05$ \\
\hline Panagiotopoulos et al. (2015) & TQ-mini & PSS & $\mathrm{PC}$ & 0.35 & $<0.000$ \\
\hline Park et al. (2017) & THI & K-BEPSI & $\mathrm{PC}$ & 0.42 & $<0.001$ \\
\hline Salviati et al. (2013) & THI & SVS & $\mathrm{SC}$ & 0.67 & $<0.000$ \\
\hline
\end{tabular}

DASS=Depression Anxiety Stress Scale, FTQ=Fear of Tinnitus Questionnaire, K-BEPSI=Korean-Brief Encounter Psychosocial Instrument, LM=Loudness Matching, MPS=Psychological Stress Measurement, $N R=$ Not Reported, $n s=$ Not Significant, $P C=$ Pearson's correlation, $P S S=$ Perceived Stress Scale, $P S Q=$ Perceived Stress Questionnaire, $P M=$ Pitch Matching, $S C=$ Spearman's correlation, SRLE=Survey of Recent Life Experiences, SVS=Stress Vulnerability Scale, TCS=Tinnitus Catastrophizing Scale, THI=Tinnitus Handicap Inventory, TFI=Tinnitus Functional Index, TQ=Tinnitus Questionnaire, VAS=Visual Analogue Scale.. 
Table 4: Studies assessed the effect of interventions on tinnitus and stress. Describing the type of the intervention and the measurements used in each study, and reporting any changes after the intervention.

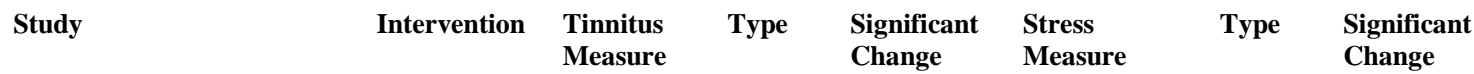

\begin{tabular}{|c|c|c|c|c|c|c|c|}
\hline Dineen et al. (1999) & $\mathrm{BBN}$ & TRQ & PsM & $\mathrm{N}$ & DGS & PsM & $\mathrm{N}$ \\
\hline Durai and Searchfield (2017) & $\mathrm{BBN}$ & $\begin{array}{l}\text { TFI \& } \\
\text { MML }\end{array}$ & $\begin{array}{l}\text { PsM \& } \\
\text { PsA }\end{array}$ & $\begin{array}{l}\text { Y \& } \\
\text { NR }\end{array}$ & DASS & PsM & $\mathrm{Y}$ \\
\hline Weise et al. (2008) & $\mathrm{BIO}$ & TQ & PsM & NR & EMG, SKC & $\mathrm{BPh}$ & NR \\
\hline Abbott et al. (2009) & CBT & TRQ & PsM & $\mathrm{N}$ & DASS & PsM & $\mathrm{N}$ \\
\hline Bruggemann et al. (2018) & $\begin{array}{l}\text { CBT } \\
\text { (multimodal) }\end{array}$ & TQ & PsM & $\mathrm{Y}$ & PSQ & PsM & $\mathrm{Y}$ \\
\hline Hesser et al. (2012) & CBT, ACT & THI, TAQ & PsM & $\mathrm{Y}$ & PSS & PsM & $\mathrm{Y}$ \\
\hline Li et al. (2019) & $\begin{array}{l}\text { CBT \& } \\
\text { sound }\end{array}$ & THI & PsM & $\mathrm{Y}$ & $\begin{array}{l}\text { CORT } \\
\text { IL-2 }\end{array}$ & $\mathrm{BPh}$ & $\begin{array}{l}\mathrm{Y} \\
\mathrm{N}\end{array}$ \\
\hline Haussler et al. (2019) & $\mathrm{CI}$ & TQ & PsM & Y & PSQ & PsM & $\mathrm{Y}(\mathrm{ns})$ \\
\hline Ketterer et al. (2018) & $\mathrm{CI}$ & TQ & PsM & Y & PSQ & PsM & M \\
\hline Olze et al. (2011a) & $\mathrm{CI}$ & TQ & PsM & $\mathrm{Y}$ & PSQ & PsM & $\mathrm{Y}$ \\
\hline Olze et al. (2011b) & $\mathrm{CI}$ & TQ & PsM & $\mathrm{Y}$ & PSQ & PsM & $\mathrm{Y}$ \\
\hline Olze et al. (2012) & $\mathrm{CI}$ & TQ & PsM & $\mathrm{Y}$ & PSQ & PsM & $\mathrm{Y}$ \\
\hline Frachet et al. (2017) & MedT & THI & PsM & $\mathrm{Y}$ & MPS & PsM & $\mathrm{Y}$ \\
\hline Aoki et al (2012) & MedT & THI & PsM & $\mathrm{N}$ & CORT/DHEA & $\mathrm{BPh}$ & $\mathrm{Y}$ \\
\hline Attanasio et al. (2012) & Music & THI, VAS & PsM & $\mathrm{Y}$ & MSP & PsM & $\mathrm{N}$ \\
\hline Kirsch \& Blanchard (1987) & PMR \& BIO & $\begin{array}{l}\text { Weekly } \\
\text { tinnitus } \\
\text { disturbanc } \\
\text { e, LM, } \\
\text { MML }\end{array}$ & PsM, & M & $\begin{array}{l}\text { EMG, } \\
\text { Reported }\end{array}$ & $\begin{array}{l}\text { BPh \& } \\
\text { PsM }\end{array}$ & M \\
\hline Weber et al. (2002) & PMR & TQ & PsM & $\mathrm{Y}$ & $\begin{array}{l}\text { PSQ, } \\
\text { Immuno- } \\
\text { biomarkers }\end{array}$ & $\begin{array}{l}\text { PsM, } \\
\mathrm{BPh}\end{array}$ & $\mathrm{Y}$ \\
\hline Bruggemann et al. (2019) & $\begin{array}{l}\text { TRT } \\
\text { (multimodal) }\end{array}$ & TQ & PsM & $\mathrm{Y}$ & PSQ & PsM & $\mathrm{Y}$ \\
\hline Crocetti et al. (2018) & TRT & THI & PsM & $\mathrm{Y}$ & $\begin{array}{l}\text { EMG, SKC, } \\
\text { HRV }\end{array}$ & $\mathrm{BPh}$ & $\mathrm{N}$ \\
\hline Seydel et al. (2010) & TRT & TQ \& & PsM \& & Y \& & PSQ & PsM & $\mathrm{Y}$ \\
\hline & & PM,LM & PsA & NR & & & \\
\hline Ylikoski et al. (2017) & tVNS & $\begin{array}{l}\text { THI, mini- } \\
\text { TQ }\end{array}$ & PsM & NR & HRV & $\mathrm{BPh}$ & Y \\
\hline Koksoy et al. (2017) & Yoga & THQ & PsM & $\mathrm{Y}$ & SSS & PsM & $\mathrm{Y}$ \\
\hline
\end{tabular}

ACT=Acceptance and Commitment Therapy, BBN=Broadband Noise, BIO=Biofeedback, BPh=Biophysiological measurement, $C B T=$ Cognitive Behavioural Therapy, $C I=$ Cochlear Implants, CORT=Cortisol,

CORT/DHEA = cortisol/ dehydroepiandrosterone ratio, DASS=Depression Anxiety Stress Scale, DGS=Derogatis Stress Profile, EMG= Electromyogram, HRV=Heart Rate Variability, LM=Loudness Matching, M=Mixed, MedT=Medical treatment, MML= Minimum Masking Level, MSP=Measure du Stress Psychologique Questionnaire, $N=N o$, ns=not significant, NR=Not Reported, $P M=$ Pitch Matching, PMR=Progressive Muscle Relaxation, PsA=Psychoacoustic measurement, PsM=Psychometric measurement, PSS=Perceived Stress Scale, $P S Q=$ Perceived Stress Questionnaire, SKC=Skin Conductance, SSS=Stress Symptoms Scale, TAQ=Tinnitus Acceptance Questionnaire, TFI=Tinnitus Functional Index, THI=Tinnitus Handicap Inventory, THQ=Tinnitus Handicap Questionnaire, TQ=Tinnitus Questionnaire, TRQ=Tinnitus Reaction Questionnaire, TRT=Tinnitus Retaining Therapy, $t$ VNS=transcutaneous vagal nerve stimulation, VAS=Visual Analogue Scale, Y=Yes. 